\title{
Diagnostic imaging of malignant insulinoma in a dog
}

\author{
Jihye Choi ${ }^{1, *}$, Seoyeon Keh ${ }^{2}$, Sungsoo Kim ${ }^{2}$, Su-Hyung Lee ${ }^{3}$, Hyejin Kim ${ }^{2}$, Heeyeon Choi ${ }^{2}$, \\ Younji Lim², Hyunwook Kim², Ahyoung Kim², Dae-yong Kim ${ }^{3}$ \\ ${ }^{1}$ Department of Veterinary Medical Imaging, College of Veterinary Medicine, Chonnam National University, \\ Gwangju 500-757, Korea \\ ${ }^{2}$ Haemaru Referral Animal Hospital, Seongnam 463-050, Korea \\ ${ }^{3}$ Department of Veterinary Pathology, College of Veterinary Medicine, Seoul National University, Seoul 151-742, Korea
}

(Received: November 11, 2011; Accepted: July 5, 2012)

\begin{abstract}
Endocrine test data from a 13-year old intact female Maltese was indicative of the presence of an insulinoma, however ultrasonography identified a pancreatic mass only after 10 months after the first admission. Following identification of both pancreatic tumor and hepatic metastasis on computed tomography (CT), surgical excision of the mass was attempted. However, total excision failed because of tumor adhesion to adjacent large vessels. The pancreatic mass was monitored over the next 25 months via ultrasonography, CT, and positron emission tomography-computed tomography (PET-CT). Histopathological and immunohistochemical data confirmed the diagnosis of insulinoma with hepatic metastasis.
\end{abstract}

Keywords : computed tomography, dog, insulinoma, positron emission tomography, ultrasonography

Diagnostic imaging in canine insulinomas can locate the lesion, define the extent of the tumor and the status of metastasis, determine respectability, and identify alternative palliative and curative treatment options [8, 10]. Canine insulinomas are often malignant and at the time of surgery, at least $45 \%$ of dogs with insulinomas already have metastases $[3,10]$. Moreover, because even small neoplastic nodules can induce clinical signs, early diagnosis and imaging methods with high sensitivity are very important for canine insulinomas [11]. Cross-sectional imaging techniques, including ultrasonography, computed tomography (CT), and magnetic resonance imaging (MRI), play major roles upon initial assessment. Functional imaging methods including nuclear scintigraphy, single-photon emission computed tomography (SPECT), and positron emission tomography-computed tomography (PET-CT), can provide information on both the primary tumor and any metastases in more detail [1]. PET$\mathrm{CT}$ is especially superior to both nuclear scintigraphy and SPECT, because this modality offers excellent sensitivity and improved spatial resolution [2]. To the best of our knowledge, however, PET-CT has not been utilized to date to evaluate canine insulinomas. Here we describe the findings of various diagnostic imaging including PET-CT examination in a dog with malignant insulinima.

A 13-year old, intact female Maltese presented with a 2week history of weakness, seizures and salivation. Hypogly- cemia (68 mg/dL; reference range: 74 143 mg/dL) and hyperinsulinemia (122.38 U/mL; reference range: $2 \sim 25 \mathrm{U} / \mathrm{mL})$ suggested that the animal's seizures were caused by an insulinoma. Radiography and ultrasonography did not reveal a lesion in any parenchymal organ including the pancreas. Seizure of this dog could not be effectively controlled with corticosteroids (prednisolone, $0.5 \mathrm{mg} / \mathrm{kg}$ per day).

On ultrasonography taken at 10 months after first admission, a homogeneous hypoechoic mass measuring about 3.1 $\mathrm{cm} \times 1.8 \mathrm{~cm}$, with smooth contours, was observed in the left pancreatic lobe and hypoechoic hepatic nodules were found in the middle and left hepatic lobes (Fig. 1). Preoperative CT examination with a slice thickness of $2.8 \mathrm{~mm}$ and a pitch of 1 revealed a poorly delineated, hypoattenuating mass (46.6 \pm 5.1 Hounsfield unit (HU)) in the left pancreatic lobe (Fig. 2). No abnormal lesion was found in the right pancreatic lobe $(50 \pm 5.1 \mathrm{HU})$. Hypoattenuating hepatic nodules were also observed. Enhanced CT images were acquired with a $50 \mathrm{sec}$ delay following injection of $3 \mathrm{mg} / \mathrm{kg}$ iohexol (Omnipaque TM; Nycomed, Ireland), at a flow rate of $1.5 \mathrm{~mL} / \mathrm{sec}$. The pancreatic mass was enhanced irregularly and measured as $2.4 \mathrm{~cm} \times 1.5 \mathrm{~cm}$. The hepatic nodules were not enhanced. In dorsal reconstruction, the portal vein close to the pancreatic lesion was compressed by the mass.

Laparotomy revealed a dark red, firm mass about $3 \mathrm{~cm}$ in diameter in the left pancreatic lobe and the remaining left

*Corresponding author

Tel: +82-62-530-2821, Fax: +82-62-530-2809

E-mail: imsono@chonnam.ac.kr 


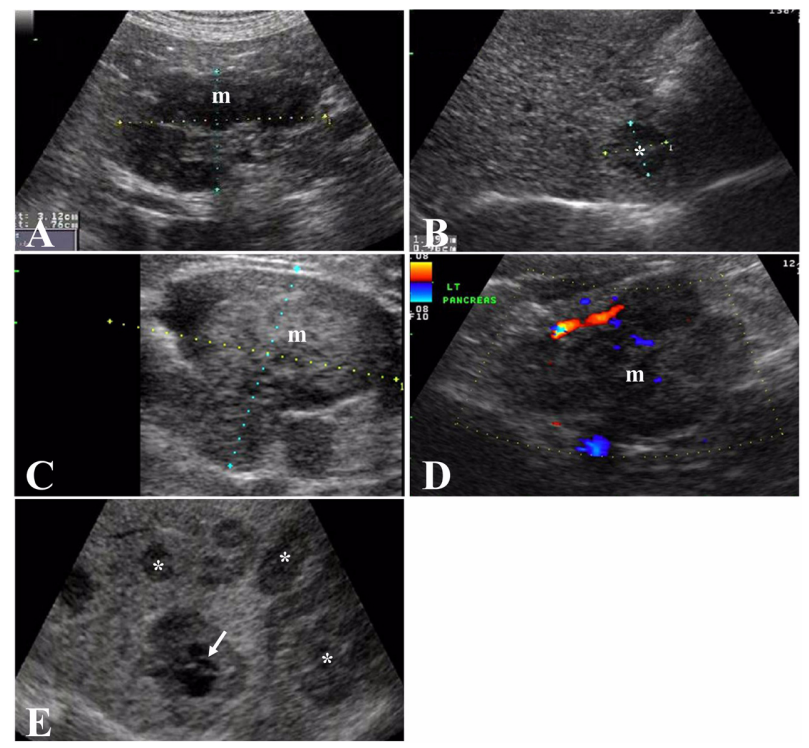

Fig. 1. Ultrasonography of a dog. (A, B) Images taken 10 months after initial admission, showing a homogeneous hypoechoic mass $(\mathrm{m})$ with a smooth contour, $3.1 \mathrm{~cm} \times 1.8 \mathrm{~cm}$ in size, in the left pancreatic lobe, along with hypoechoic nodules (*) in the left hepatic lobes. (C) About 8 months after commencement of chemotherapy, the pancreatic mass (m) increases in size to $3.3 \mathrm{~cm} \times 2.3 \mathrm{~cm}$ and invades the adjacent portal vein. (D, E) At 25 months after admission, note the similar echotexture and contour of the pancreatic mass $(\mathrm{m})$ compared with previous images and increase of hepatic nodules $\left(^{*}\right)$ in size and number (arrow).

lobe parenchyma was firmly palpated (Fig. 3). As the mass had adhered to the caudal vena cava, the portal vein, and the gastrosplenic artery, debulking and biopsy rather than total excision of the left pancreatic lobe were performed. Multiple hepatic nodules were observed and biopsied. The dog was diagnosed with malignant insulinoma and concurrent hepatic metastasis through histological and immunohistochemical examinations using antibodies against insulin and glucagon.

The dog was donated to the Haemaru Referral Animal Hospital (Korea). Chemotherapy using streptozotocin (Sigma Aldrich, USA) and serial diagnostic imaging were performed for about 13 months, until the dog was euthanized because of persistent seizures and progress of the lesions. Six months after commencement of chemotherapy, the size of the pancreatic mass had slightly decreased to $2.6 \mathrm{~cm} \times 2.3 \mathrm{~cm}$ temporarily, however increased again (to $3.3 \mathrm{~cm} \times 2.3 \mathrm{~cm}$ ) at 8 months. At this time, tumor invasion into the portal vein at a point close to the left pancreatic lobe was observed (Fig. 1). Doppler ultrasonography revealed turbulent flow near the intraluminal lesion with normal flow in the portal vein distal to the lesion. On serial ultrasonographic examinations, neither the echotexture nor the border of the pancreatic mass had changed, and only the numbers and sizes of the hepatic nodules had increased. CT examination was performed again at 10 month after first CT scanning using the same protocol

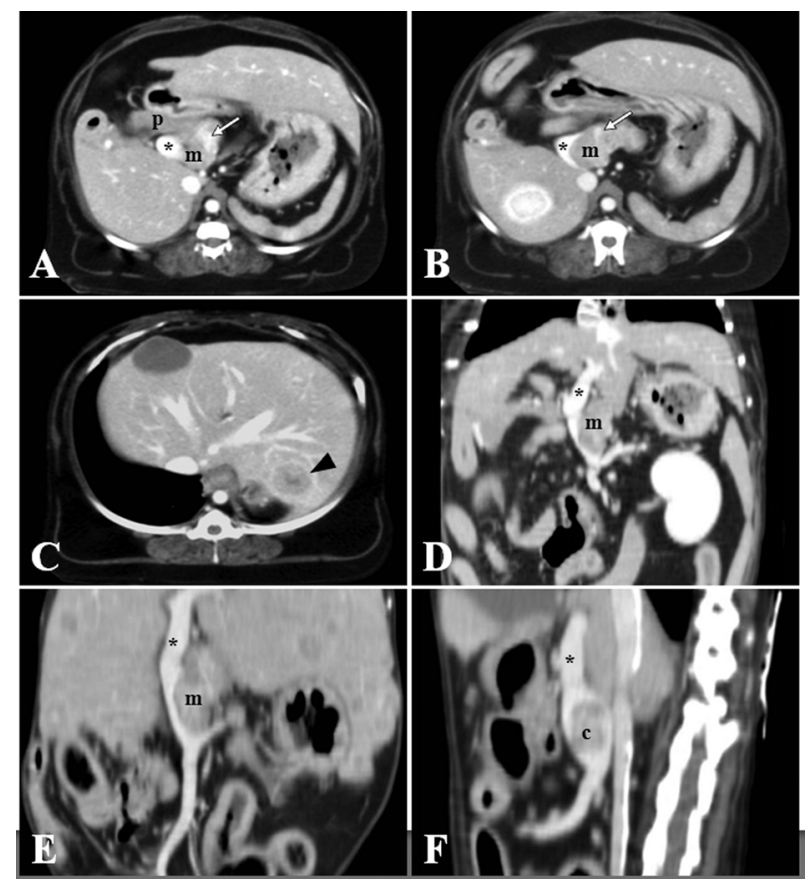

Fig. 2. Enhanced computed tomography (CT) taken at 10 months and 20 months after initial admission. In initial CT examination (A-D), a single, irregularly enhanced pancreatic mass ( $\mathrm{m})$ is found in the left pancreatic lobe (p). The mass compresses the adjacent portal vein $(*)$. Dorsal reconstruction shows the compressed portal vein $(*)$ by the pancreatic mass $(\mathrm{m})$. Liver metastases are visible as hypoattenuating lesions (arrowheads) with ring enhancement. In CT recheck, the size of pancreatic mass (m) has increased to $2.7 \mathrm{~cm} \times 1.6 \mathrm{~cm}$ in dorsal view (E). A filling defect (c) within the portal vein $(*)$ is observed in sagittal reconstruction $(\mathrm{F})$.

and parameters applied to the previous CT examination. Except slight decrease in the density of a pancreatic mass (41 $\pm 4.7 \mathrm{HU})$ and increase of the size $(2.81 \mathrm{~cm} \times 2.05 \mathrm{~cm})$ compared with previous CT finding, the number of the pancreatic mass and enhanced pattern were not changed (Fig. 2). Dorsal and sagittal reconstructions showed the invasion of portal vein by the mass with a filling defect within the vascular lumen. Multiple hepatic nodules with central hypoattenuation and peripheral enhancement were observed. PET-CT (Discovery $600 \mathrm{PET} / \mathrm{CT}$ system; GE Healthcare, USA) was conducted at $40 \mathrm{~min}$ after injection of $0.3 \mathrm{mCi} / \mathrm{kg}$ of $18-\mathrm{flu}-$ oro-2-deoxyglucose (18F-FDG) intravenously (Fig. 4). No image showed an area of increased radiopharmaceutical accumulation in the region of the pancreas. The hypoattenuating hepatic nodules had maximum standardized uptake values (SUV) of 1.2 1.3, similar to the SUV of the background hepatic parenchyma $(1.0 \sim 1.1)$.

Most islet cell tumors are too small at the time of diagnosis to detect or stage using radiography and/or ultrasonography [9]. In the dog of the present report, ultrasonography could show the pancreatic lesion only 10 months after the 


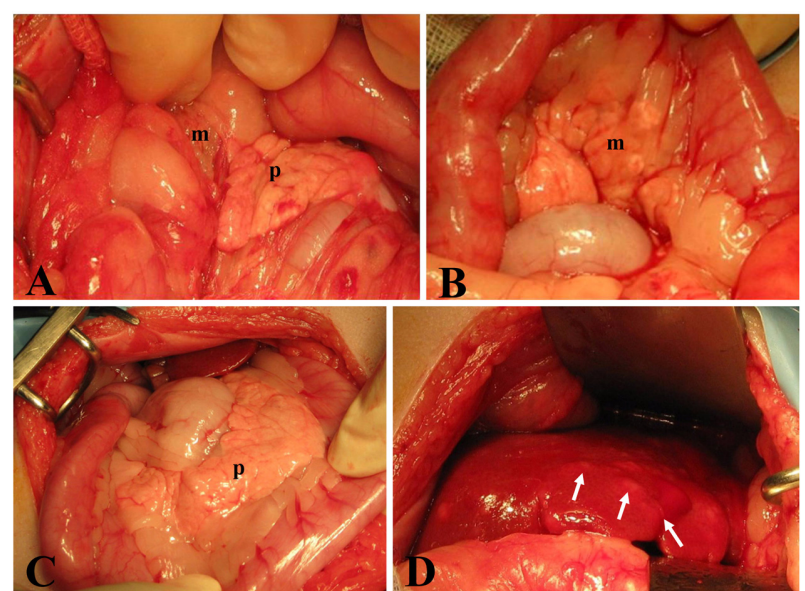

Fig. 3. (A, B) Grossly a dark red, firm mass (m) in the left pancreatic lobe (p). (C) No abnormal lesions in the right pancreatic lobe (p). (D) Multiple nodules on the hepatic lobes.

onset of clinical signs. The insulinoma appeared as a hypoechoic solitary mass with a distinct border. This ultrasonographic finding is not pathognomonic, because other pancreatic disorders may be similar in appearance. However, serial ultrasonography is accurate and useful when used to monitor the progress of pancreatic masses and metastatic lesions [9, 11]. In this dog, the echo patterns and shape of the insulinoma did not change significantly over time, however, the pancreatic mass invaded an adjacent portal vein and metastastic nodules in the liver dramatically increased in terms of both size and number.

CT has been regarded as the gold standard for examination of human pancreatic tumors [7]. CT showed similar findings with those of ultrasonography in the present case. However, the spatial relationship between the tumor and the adjacent vessel could be successfully investigated in more detail when contrast-enhanced CT and sagittal reconstruction were employed. The sensitivity of CT could not be directly compared to that of ultrasonography, because CT examination could not be performed at the early stages of insulinoma. Insulinomas are often histopathologically hypervascular, therefore $\mathrm{CT}$ images obtained during the arterial phase can show clearly delineated enhancing tumor lesions $[4,5]$. Although dynamic CT examination was not used in this study, a focal region showing contrast enhanced changes within the pancreatic mass was identified, thus exhibiting the hypervascular characteristics of insulinoma.

A glucose-derivative, [18]F-FDG, the most frequently used radiopharmaceutical was applied. PET-CT failed to provide useful information for detecting the primary tumor or identifying the extent of the metastatic lesions in this case, because the employment of PET-CT did not detect the pancreatic mass, and the SUV of the hepatic lesion was similar to that of the normal hepatic parenchyma. The limited utility of FDG PET-CT in detection of canine insulinomas may be attributable to the low level of glucose turnover [6]. How-
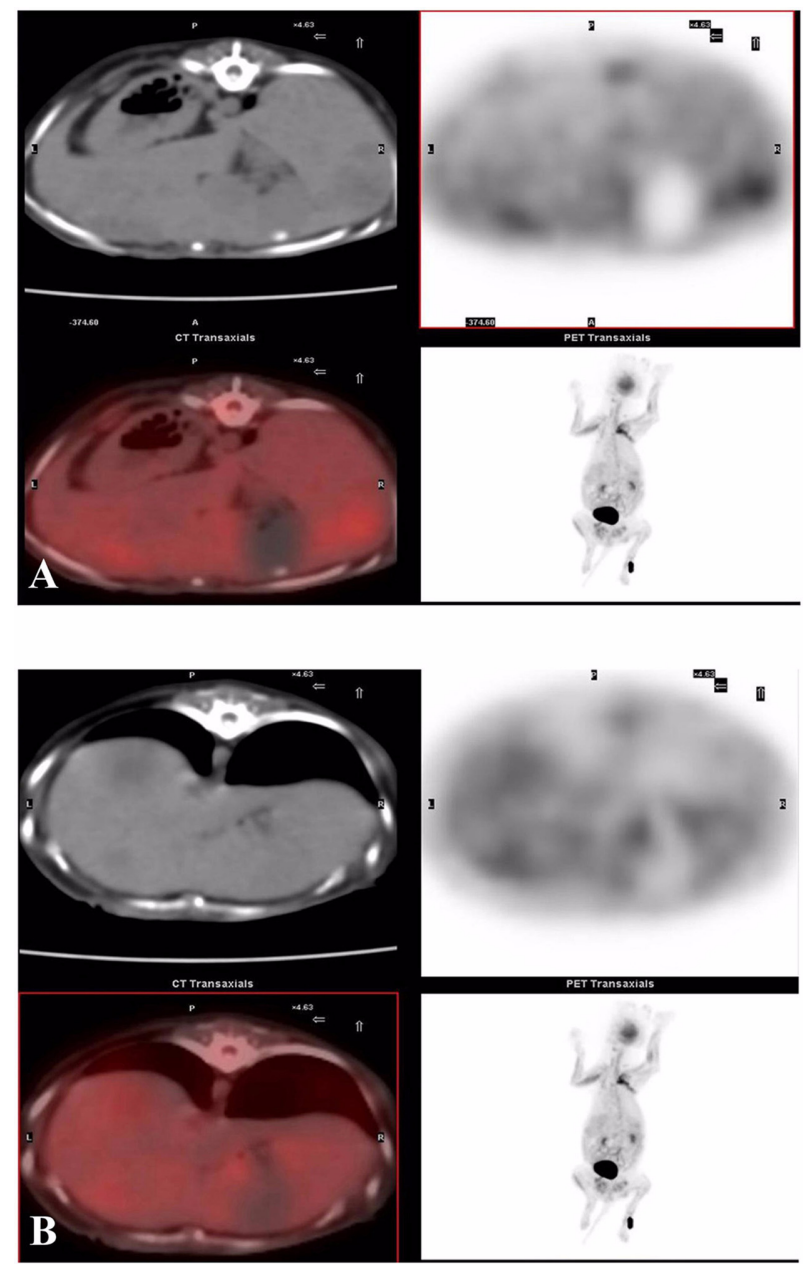

Fig. 4. Positron emission tomography-computed tomography (PET-CT) using 18-fluoro-2-deoxyglucose ([18]F-FDG). No marked change of pancreatic mass and hepatic nodules is detected.

ever PET-CT using [18]F-FDG was applied to only one case, therefore further studies about FDG PET-CT examination in canine insulinomas are needed including time activity curve and dose-response study of [18]F-FDG to establish the optimal scan condition for detecting canine insulinomas.

In the present study, various diagnostic imaging modalities were applied to study malignant insulinoma in a dog. The insulinoma exhibited vascular invasion and hepatic metastasis; however, none of the size, shape, or characteristics of the pancreatic mass changed significantly over time. PET-CT images using [18]F-FDG could not detect either the primary pancreatic tumor or hepatic metastases in this case, however first application of PET-CT examination in canine insulinoma was attempted and suggested the need of further study about functional diagnostic imaging in this tumor.

\section{References}

1. Bhate K, Mok WY, Tran K, Khan S, Al-Nahhas A. 
Functional assessment in the multimodality imaging of pancreatic neuro-endocrine tumours. Minerva Endocrinol 2010, 35, 17-25.

2. Brom M, Oyen WJG, Joosten L, Gotthardt M, Boerman OC. ${ }^{68} \mathrm{Ga}$-labelled exendin-3, a new agent for the detection of insulinomas with PET. Eur J Nucl Med Mol Imaging 2010, 37, 1345-1355.

3. Caywood DD, Klausner JS, O'Leary TP, Withrow SJ, Richardson RC, Harvey HJ, Norris AM, Henderson RA, Johnston SD. Pancreatic insulin-secreting neoplasms: clinical, diagnostic, and prognostic features in 73 dogs. J Am Anim Hosp Assoc 1988, 24, 577-584.

4. Gritzmann N, Macheiner P, Hollerweger A, Hübner E. $\mathrm{CT}$ in the differentiation of pancreatic neoplasms--progress report. Dig Dis 2004, 22, 6-17.

5. Iseri T, Yamada K, Chijiwa K, Nishimura R, Matsunaga S, Fujiwara R, Sasaki N. Dynamic computed tomography of the pancreas in normal dogs and in a dog with pancreatic insulinoma. Vet Radiol Ultrasound 2007, 48, 328-331.
6. Kauhanen S, Seppänen M, Minn H, Nuutila P. Clinical PET imaging of insulinoma and beta-cell hyperplasia. Curr Pharm Des 2010, 16, 1550-1560.

7. Kim T, Murakami T, Takahashi S, Okada A, Hori M, Narumi Y, Nakamura H. Pancreatic CT imaging: effects of different injection rates and doses of contrast material. Radiology 1999, 212, 219-225.

8. Kruth SA, Feldman EC, Kennedy PC. Insulin-secreting islet cell tumors: establishing a diagnosis and the clinical course for 25 dogs. J Am Vet Med Assoc 1982, 181, 5458.

9. Lamb CR, Simpson KW, Boswood A, Matthewman LA. Ultrasonography of pancreatic neoplasia in the dog: a retrospective review of 16 cases. Vet Rec 1995, 137, 65-68.

10. Lester NV, Newell SM, Hill RC, Lanz OI. Scintigraphic diagnosis of insulinoma in a dog. Vet Radiol Ultrasound 1999, 40, 174-178.

11. Madarame H, Kayanuma H, Shida T, Tsuchiya $\mathbf{R}$. Retrospective study of canine insulinomas: eight cases (2005-2008). J Vet Med Sci 2009, 71, 905-911. 